\title{
Measurement-induced chaos and quantum state discrimination in an iterated Tavis-Cummings scheme
}

\author{
Juan Mauricio Torres, ${ }^{1,2}$ József Zsolt Bernád, ${ }^{1}$ Gernot Alber, ${ }^{1}$ Orsolya Kálmán, ${ }^{3}$ and Tamás Kiss ${ }^{3}$ \\ ${ }^{1}$ Institut für Angewandte Physik, Technische Universität Darmstadt, D-64289, Germany \\ ${ }^{2}$ Instituto de Física, Benemérita Universidad Autónoma de Puebla, Apdo. Postal J-48, Puebla, Pue. 72570, México \\ ${ }^{3}$ Institute for Solid State Physics and Optics, Wigner Research Centre, Hungarian Academy of Sciences, P.O. Box 49, \\ H-1525 Budapest, Hungary
}

(Received 7 October 2016; published 14 February 2017)

\begin{abstract}
A cavity quantum electrodynamical scenario is proposed for implementing a Schrödinger microscope capable of amplifying differences between nonorthogonal atomic quantum states. The scheme involves an ensemble of identically prepared two-level atoms interacting pairwise with a single mode of the radiation field as described by the Tavis-Cummings model. By repeated measurements of the cavity field and of one atom within each pair a measurement-induced nonlinear quantum transformation of the relevant atomic states can be realized. The intricate dynamical properties of this nonlinear quantum transformation, which exhibits measurement-induced chaos, allow approximate orthogonalization of atomic states by purification after a few iterations of the protocol and thus the application of the scheme for quantum state discrimination.
\end{abstract}

DOI: 10.1103/PhysRevA.95.023828

\section{INTRODUCTION}

Consistent with the no-cloning theorem, nonorthogonal quantum states cannot be distinguished perfectly. However, for purposes of quantum communication, for example, it is necessary to be able to distinguish between two informationcarrying quantum states even if they have become nonorthogonal after passing through a channel. Therefore, quantum processes capable of distinguishing between nonorthogonal quantum states in an optimal way offer interesting perspectives for applications in quantum information science. Prominent examples of such processes are the Helstrom measurement [1], which minimizes errors, and the Ivanovic-Dieks-Peres measurement [2-4], which distinguishes pure quantum states in an unambiguous way.

Alternatively, nonorthogonal quantum states can also be distinguished with the help of nonlinear quantum state transformations [5]. Quantum state purification protocols [6-10] are early examples of such nonlinear quantum state transformations. Thereby, identically prepared quantum systems are subjected to an entangling unitary transformation and a subsequent selective measurement performed on parts of the system. Iterating these operations typically results in a strong dependence of the final state on the initial conditions and in measurement-induced complex chaos [11,12]. Recently, it has been demonstrated [13] that the resulting strong sensitivity to initial conditions can, in principle, be used to amplify small initial differences of quantum states, thus realizing a Schrödinger microscope, a term originally suggested by Lloyd and Slotine [14], capable of distinguishing nonorthogonal quantum states. Although Helstrom and Ivanov-Dieks-Peres measurements have already been realized experimentally both optically [15,16] and in the solid state [17], a Schrödinger microscope based on nonlinear quantum state transformations has not yet been realized.

Motivated by these developments the purpose of this paper is twofold, namely, to propose an experimental scenario in which iterated nonlinear dynamics can be realized with atomic qubits and to explore the characteristic features of the underlying nonlinear quantum state transformation in order to present a Schrödinger microscope and to demonstrate its applicability for quantum state discrimination. In view of its possibilities to measure and control the interaction of individual atoms with a single mode of the quantized radiation field with high precision, the area of cavity quantum electrodynamics offers interesting perspectives for future experimental implementations in this direction $[18,19]$. Inspired by recent experimental advances which realize the Tavis-Cummings model [19], in our proposal an ensemble of identically prepared two-level atoms (qubits) which interact pairwise with a single mode of the radiation field is considered. Afterwards, one member of each pair and the corresponding cavity field are measured. Conditioned on these measurement results, the unmeasured atoms are kept or discarded. In practice, this may be implemented with the help of a single cavity and a pair of optical conveyor belts [18], for example. Subsequently, the atoms are moved through the cavity by the conveyor belts in such a way that only one pair of atoms interacts with the cavity mode at a time, and then the cavity is reinitialized after each interaction. The remaining atoms form a new identically prepared ensemble of smaller size. Like in entanglement distillation protocols, the state changes of the remaining two-level atoms are described by an iterated nonlinear quantum transformation.

We analyze the emerging nonlinear quantum state transformation and show that it exhibits measurement-induced complex chaos. We characterize the different parameter regimes, the possible stable fixed points and fixed cycles of the dynamics, and the regions of convergence as well as nonconverging sets of initial states, forming the so-called Julia set. Based on this analysis we identify a case where the two stable fixed points correspond to orthogonal quantum states of the atom and the Julia set forms a line, separating the two regions of stability. Here the system can be utilized as a Schrödinger microscope capable of amplifying the distinguishability of nonorthogonal 
quantum states. In the presented setting, two-level atoms with small excitation amplitudes can be discriminated according to the sign of the real part of their excitation amplitudes. Thus it is also suitable to discriminate noisy nonorthogonal quantum states.

This paper is organized as follows. In Sec. II the dynamical equations of the two-atom Tavis-Cummings model are solved, and exact and approximate analytical solutions are presented, facilitating our subsequent treatment. Furthermore, the atomic postselection scheme is discussed; this scheme is used in Sec. III to discuss our protocol for implementing a nonlinear map of atomic probability amplitudes. In Sec. IV the fractal structure of the resulting nonlinear map is analyzed. In Sec. V our proposal for implementing a Schrödinger microscope is presented. Finally, in Sec. VI some aspects concerning possible experimental realizations of our proposal with current technology are discussed.

\section{THE TWO-ATOM TAVIS-CUMMINGS MODEL}

The two-atom Tavis-Cummings model describes the resonant interaction between two atoms, say $A$ and $B$, and a single mode of the radiation field [20]. The atoms have ground states $|0\rangle_{i}$ and excited states $|1\rangle_{i}(i \in\{A, B\})$ separated by an energy difference of $\hbar \omega$ which matches the energy of a photon inside the empty cavity. In the interaction picture the Hamiltonian can be expressed in the following form:

$$
\hat{H}=\hbar g \sum_{i=A, B}\left(\hat{\sigma}_{i}^{+} \hat{a}+\hat{\sigma}_{i}^{-} \hat{a}^{\dagger}\right),
$$

where $\hat{\sigma}_{i}^{+}=|1\rangle\left\langle\left. 0\right|_{i}\right.$ and $\left.\hat{\sigma}_{i}^{-}=\mid 0\right\rangle\left\langle\left. 1\right|_{i}\right.$ are the atomic raising and lowering operators $(i \in\{A, B\})$ and $\hat{a}\left(\hat{a}^{\dagger}\right)$ is the annihilation (creation) operator of the single-mode field. The interaction picture is taken with respect to the reference Hamiltonian

$$
\hat{H}_{0}=\hbar \omega\left(\hat{a}^{\dagger} \hat{a}+|1\rangle\left\langle\left. 1\right|_{A}+\mid 1\right\rangle\left\langle\left. 1\right|_{B}\right),\right.
$$

which is a constant of motion as it commutes with the interaction Hamiltonian $\hat{H}$. For this reason both operators, $\hat{H}$ and $\hat{H}_{0}$, can be diagonalized simultaneously. In fact, there is a set of common eigenvectors with eigenvalue zero, namely, $\left\{\left|\Psi^{-}\right\rangle|n\rangle\right\}_{n=0}^{\infty}$. These states are written in terms of the Fock states $|n\rangle$ of the field and the atomic states $|i, j\rangle=|i\rangle_{A}|j\rangle_{B}$ $(i, j \in\{0,1\})$ together with the atomic Bell states

$$
\left|\Psi^{ \pm}\right\rangle=\frac{1}{\sqrt{2}}(|0,1\rangle \pm|1,0\rangle) .
$$

The evaluation of the rest of the eigenvectors can be simplified by realizing that $\hat{H}$ has a block-diagonal form in the basis

$$
\begin{aligned}
& \{|0,0\rangle|0\rangle\} \oplus\left\{\left|\Psi^{+}\right\rangle|0\rangle,|0,0\rangle|1\rangle\right\} \\
& \quad \oplus\left\{|1,1\rangle|n-2\rangle,\left|\Psi^{+}\right\rangle|n-1\rangle,|0,0\rangle|n\rangle\right\}_{n=2}^{\infty},
\end{aligned}
$$

with blocks given by the following matrices:

$$
\begin{aligned}
H^{(0)} & =0, \quad H^{(1)}=\hbar g\left(\begin{array}{cc}
0 & \sqrt{2} \\
\sqrt{2} & 0
\end{array}\right), \\
H^{(n \geqslant 2)} & =\hbar g\left(\begin{array}{ccc}
0 & \sqrt{2(n-1)} & 0 \\
\sqrt{2(n-1)} & 0 & \sqrt{2 n} \\
0 & \sqrt{2 n} & 0
\end{array}\right) .
\end{aligned}
$$

The eigenvalues of these matrices are given by $\{0\}$ for $n=0$, $\{-\sqrt{2} \hbar g, \sqrt{2} \hbar g\}$ for $n=1$, and $\left\{0,-\hbar \omega_{n}, \hbar \omega_{n}\right\}$ for $n \geqslant 2$, with

$$
\omega_{n}=g \sqrt{4 n-2} .
$$

The transformations that diagonalize each of the blocks $H^{(n)}$ are given by

$$
\begin{aligned}
O^{(1)} & =\frac{1}{\sqrt{2}}\left(\begin{array}{rr}
1 & 1 \\
-1 & 1
\end{array}\right), \\
O^{(n \geqslant 2)} & =\frac{1}{\sqrt{4 n-2}}\left(\begin{array}{ccc}
-\sqrt{2 n} & \sqrt{n-1} & \sqrt{n-1} \\
0 & -\sqrt{2 n-1} & \sqrt{2 n-1} \\
\sqrt{2 n-2} & \sqrt{n} & \sqrt{n}
\end{array}\right) .
\end{aligned}
$$

These matrices are the blocks of the orthogonal transformation $\hat{O}$ that diagonalizes the Hamiltonian $\hat{H}$ as $\hat{O}^{\dagger} \hat{H} \hat{O}$.

\section{A. Exact solution}

Having solved the eigenvalue problem for $\hat{H}$, it is now possible to evaluate the time-dependent state vector

$$
\left|\Psi_{t}\right\rangle=e^{-i \hat{H} t / \hbar}\left|\Psi_{0}\right\rangle
$$

for any given initial pure state $\left|\Psi_{0}\right\rangle$. In this work we consider as the initial condition a normalized product state of the two atoms and the single-mode field that can be expressed as

$$
\begin{aligned}
\left|\Psi_{0}\right\rangle & =\left|\Psi_{0}^{\text {at }}\right\rangle|\alpha\rangle, \\
\left|\Psi_{0}^{\text {at }}\right\rangle & =c_{0}|0,0\rangle+c_{-}\left|\Psi^{-}\right\rangle+c_{+}\left|\Psi^{+}\right\rangle+c_{1}|1,1\rangle .
\end{aligned}
$$

We have considered a general pure state $\left|\Psi_{0}^{\text {at }}\right\rangle$ of the atoms with probability amplitudes $c_{ \pm}, c_{0}$, and $c_{1}$. For the single mode of the radiation field we have chosen a coherent state

$$
|\alpha\rangle=\sum_{n=0}^{\infty} e^{-\frac{|\alpha|^{2}}{2}} \frac{\alpha^{n}}{\sqrt{n !}}|n\rangle, \quad \alpha=\sqrt{\bar{n}} e^{i \phi},
$$

with mean photon number $\bar{n}$. Using the eigenbasis of $\hat{H}$, the exact solution of the time-dependent state vector can be written as

$$
\left|\Psi_{t}\right\rangle=|0,0\rangle\left|\chi_{t}^{-1}\right\rangle+\left|\Psi^{+}\right\rangle\left|\chi_{t}^{0}\right\rangle+|1,1\rangle\left|\chi_{t}^{1}\right\rangle+c_{-}\left|\Psi^{-}\right\rangle|\alpha\rangle,
$$

with the relevant photonic states

$$
\begin{aligned}
\left|\chi_{t}^{-1}\right\rangle & =c_{0} p_{0}|0\rangle+\sum_{n=1}^{\infty} \frac{\sqrt{n}\left(\xi_{n, t}^{-}-\xi_{n, t}^{+}\right)+\sqrt{n-1} \xi_{n}}{\sqrt{2 n-1}}|n\rangle, \\
\left|\chi_{t}^{0}\right\rangle & =\sum_{n=1}^{\infty}\left(\xi_{n, t}^{-}+\xi_{n, t}^{+}\right)|n-1\rangle \\
\left|\chi_{t}^{1}\right\rangle & =\sum_{n=2}^{\infty} \frac{\sqrt{n-1}\left(\xi_{n, t}^{-}-\xi_{n, t}^{+}\right)-\sqrt{n} \xi_{n}}{\sqrt{2 n-1}}|n-2\rangle
\end{aligned}
$$

and with the aid of the following abbreviations:

$$
\begin{aligned}
\xi_{n, t}^{ \pm} & =\frac{e^{ \pm i \omega_{n} t}}{2}\left(c_{+} \mp \frac{c_{0} p_{n}+\sqrt{n-1} c_{1} p_{n-2}}{\sqrt{2 n-1}}\right), \\
\xi_{n} & =\frac{\sqrt{n-1} c_{0} p_{n}-\sqrt{n} c_{1} p_{n-2}}{\sqrt{2 n-1}}, \quad p_{n}=\alpha^{n} \sqrt{e^{-|\alpha|^{2}} / n !} .
\end{aligned}
$$




\section{B. Coherent-state approximation}

The time-dependent solution of the state vector can be significantly simplified in the case of high values of the mean photon number, i.e., $\bar{n} \gg 1$. In this limit $\bar{n} \gg \sqrt{\bar{n}}$; that is, the mean of the Poisson distribution $\bar{n}$ is much larger than the standard deviation $\sqrt{\bar{n}}$. Therefore we approximate $\sqrt{(n-1) /(2 n-1)}$ and $\sqrt{n /(2 n-1)}$ by $1 / \sqrt{2}$, and we also use the approximations

$$
\begin{gathered}
p_{n}=\sqrt{\frac{\bar{n}}{n} e^{i \phi} p_{n-1} \approx e^{i \phi} p_{n-1},} \\
\omega_{n} / g \approx \sqrt{4 \bar{n}+2}+2 \frac{n-\bar{n}-1}{\sqrt{4 \bar{n}+2}} .
\end{gathered}
$$

The last line is obtained from the first-order Taylor expansion in $n$ of the frequencies around $\bar{n}+1$. This is valid whenever the product of the second-order contribution times the interaction time $t$ remains small, a condition that is satisfied when $g t \ll \bar{n}$ $[21,22]$. With these considerations and by introducing the abbreviations

$$
\eta_{ \pm}=\frac{1}{2}\left(c_{+} \mp d_{\phi}^{+}\right), \quad d_{\phi}^{ \pm}=\frac{e^{i \phi} c_{0} \pm e^{-i \phi} c_{1}}{\sqrt{2}},
$$

the photonic states can be simplified to

$$
\begin{aligned}
\left|\chi_{t}^{k}\right\rangle & \approx \frac{e^{i k \phi}}{\sqrt{1+|k|}}\left[\eta_{-}\left|F_{k, t}^{-}\right\rangle+(-1)^{k} \eta_{+}\left|F_{k, t}^{+}\right\rangle-k d_{\phi}^{-}|\alpha\rangle\right] \\
k & \in\{-1,0,1\}
\end{aligned}
$$

where we have introduced the field states

$$
\left|F_{k, t}^{ \pm}\right\rangle=e^{ \pm i 2 g t \frac{1+k(\bar{n}+1)}{\sqrt{4 \bar{n}+1}}}\left|\alpha e^{\frac{ \pm i 2 g t}{\sqrt{4 \bar{n}+1}}}\right\rangle, \quad k \in\{-1,0,1\},
$$

which are coherent states up to an additional phase.

\section{Atomic postselection}

The description in terms of coherent states allows a simpler analysis of the dynamics. Our aim is to prepare the atoms in an atomic postselection scenario where the atoms are prepared conditioned on a successful projection of the field onto the initial coherent state $|\alpha\rangle$ in a simplified and ideal implementation. In such a case, one would have to consider the following overlaps:

$$
\left\langle\alpha \mid \chi_{t}^{k}\right\rangle \approx-k e^{i k \phi} \frac{e^{i \phi} c_{0} \pm e^{-i \phi} c_{1}}{2}
$$

This result can be obtained by noting that the overlap between coherent states is given by

$$
\left|\left\langle\alpha \mid \alpha e^{\frac{ \pm i 2 g t}{\sqrt{4 \bar{n}+1}}}\right\rangle\right|=\left|\exp \left[-\bar{n}\left(1-e^{\frac{ \pm i 2 g t}{\sqrt{4 \bar{n}+1}}}\right)\right]\right| \approx e^{-g^{2} t^{2}},
$$

which can be neglected if $g t \gg 1$. Therefore, after the interaction with the resonator and projection onto state $|\alpha\rangle$, both atoms are left in the state

$$
\frac{c_{-}}{Q_{1}}\left|\Psi^{-}\right\rangle+\frac{e^{i \phi} c_{0}-e^{-i \phi} c_{1}}{2 Q_{1}}\left(e^{-i \phi}|0,0\rangle-e^{i \phi}|1,1\rangle\right),
$$

with $Q_{1}^{2}=\left|c_{1}\right|^{2}+\left|e^{i \phi} c_{0}-e^{-i \phi} c_{1}\right|^{2} / 2$ being the success probability. The final state is actually a superposition of two states with probability amplitudes proportional to the initial ones. Therefore the atomic postselection can be understood as a projection of the atomic state with the following rank-two projector:

$$
\hat{M}=\left|\Psi^{-}\right\rangle\left\langle\Psi^{-}|+| \Phi_{\phi}^{-}\right\rangle\left\langle\Phi_{\phi}^{-}\right|,
$$

where we have introduced the state $\left|\Phi_{\phi}^{-}\right\rangle=$ $\left(e^{-i \phi}|0,0\rangle-e^{i \phi}|1,1\rangle\right) / \sqrt{2}$. The operation $\hat{M}$ represents the effective description of the interaction of the atoms with the resonator and the postselection via measurement of the field.

\section{Atomic postselection by balanced homodyne detection}

Considering the projection onto a coherent state is an idealization that provides a convenient simplified picture. In practice, however, it is sufficient to project onto a state with vanishing overlap with the time-dependent field components $\left|F_{k, t}^{ \pm}\right\rangle$and with finite overlap with $|\alpha\rangle$. A typical experimental setting able to achieve this goal is a balanced homodyne measurement [21]. The basic idea is to use a $50: 50$ beam splitter to combine the field to be measured with a reference coherent field parametrized by its phase $\theta$. Photons from the two outputs of the beam splitter are collected using photodetectors. In the strong limit of the reference field and assuming ideal photodetectors [23], the probability of measuring a photocurrent difference between the detectors is proportional to the projection of the field onto the eigenstate $\left|q_{\theta}\right\rangle$ of a field quadrature $\hat{q}_{\theta}=\left(\hat{a} e^{-i \theta}+\hat{a}^{\dagger} e^{i \theta}\right) / \sqrt{2}$. This probability density for a coherent state $|\alpha\rangle$ is given by

$$
\left|\left\langle q_{\theta} \mid \alpha\right\rangle\right|^{2}=\frac{1}{\sqrt{\pi}} \exp \left\{-\left[q_{\theta}-\tilde{q}_{\theta}\right]^{2}\right\},
$$

with $\tilde{q}_{\theta}=\left(\alpha e^{-i \theta}+\alpha^{*} e^{i \theta}\right) / \sqrt{2}$. This overlap can approach its maximum value by choosing the phase in such a way that $\tilde{q}_{\theta}=0$ and restricting values of $q_{\theta}$ close to zero. The square of the overlap with the other field components, which are also coherent states, can be evaluated as

$$
\left|\left\langle q_{\theta} \mid F_{k m t}^{ \pm}\right\rangle\right|^{2}=\frac{1}{\sqrt{\pi}} \exp \left\{-\left[q_{\theta}-\tilde{q}_{\Theta_{t}^{ \pm}}\right]^{2}\right\},
$$

with $\Theta_{t}^{ \pm}=\theta \mp 2 g t / \sqrt{4 \bar{n}+1}$. By choosing an appropriate interaction time $t$, these overlaps can be made exponentially small.

\section{A NONLINEAR MAP OF PURE ATOMIC STATES}

In this section we use the atomic postselection scheme of the two-atom Tavis-Cummings model in order to implement an entangling quantum operation which by iteration leads to a nonlinear mapping of atomic probability amplitudes. The protocol is depicted schematically in Fig. 1. We consider the two two-level atoms initially prepared in a product state of the form $(z \in \mathbb{C})$

$$
\left|\Psi_{0}^{\mathrm{at}}\right\rangle=\left|\psi_{0}\right\rangle_{A} \otimes\left|\psi_{0}\right\rangle_{B}, \quad\left|\psi_{0}\right\rangle=\frac{|0\rangle+z e^{i \phi}|1\rangle}{\sqrt{1+|z|^{2}}} .
$$

For later convenience we have included the phase $\phi$ of the coherent state. Before interacting with the optical resonator, a unitary gate $\hat{U}_{\varphi}^{B}$ is applied to atom $B$. We choose the following 


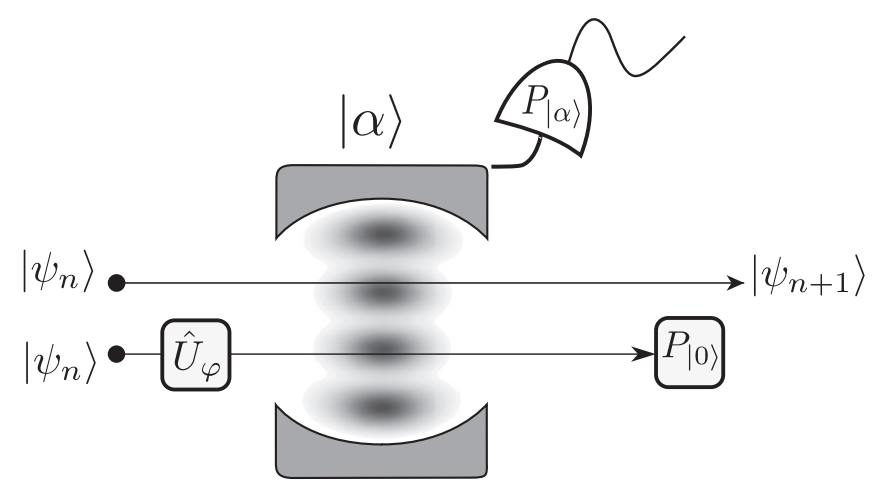

FIG. 1. Two two-level atoms in the same state $\left|\psi_{n}\right\rangle$ interact with the cavity field prepared in a coherent state $|\alpha\rangle$. Before the interaction, the gate $\hat{U}_{\varphi}$ is applied to one of the atoms, and after the interaction and the projection of the field onto the initial coherent state, this same atom is projected onto its ground state. Finally, the other atom is left in the state $\left|\psi_{n+1}\right\rangle$.

gate:

$$
\hat{U}_{\varphi}=\left(\begin{array}{cc}
e^{i \varphi} & 0 \\
0 & -e^{-i \varphi}
\end{array}\right),
$$

which can be implemented by driving the atomic transition with a resonant classical electromagnetic field and properly controlling the coupling and duration of the interaction [24-26]. After the application of $\hat{U}_{\varphi}^{B}$ and before entering the resonator we get the following atomic probability amplitudes:

$$
\begin{aligned}
c_{0} & =\left\langle 0,0\left|\hat{U}_{\varphi}^{B}\right| \Psi_{0}^{\mathrm{at}}\right\rangle=-e^{-i \varphi} /\left(1+|z|^{2}\right), \\
c_{1} & =\left\langle 1,1\left|\hat{U}_{\varphi}^{B}\right| \Psi_{0}^{\mathrm{at}}\right\rangle=z^{2} e^{i(\varphi-2 \phi)} /\left(1+|z|^{2}\right), \\
c_{-} & =\left\langle\Psi^{-}\left|\hat{U}_{\varphi}^{B}\right| \Psi_{0}^{\mathrm{at}}\right\rangle=\sqrt{2} z e^{i \phi} \cos \varphi /\left(1+|z|^{2}\right) .
\end{aligned}
$$

The probability amplitude $c_{+}$does not need to be specified, as the resulting quantum operation projects the atoms onto a subspace orthogonal to $\left|\Psi^{+}\right\rangle$, as can be noted from Eq. (19). With these initial conditions, both atoms interact with the electromagnetic field inside a cavity prepared in a coherent state $|\alpha\rangle$. After the interaction a projection $P_{|\alpha\rangle}$ of the field onto the initial coherent state $|\alpha\rangle$ is performed, and the atoms are left in the state

$$
\frac{\sqrt{2} z e^{i \phi} \cos \varphi}{\left(1+|z|^{2}\right) Q_{1}}\left|\Psi^{-}\right\rangle-\frac{e^{-i \varphi}+z^{2} e^{i \varphi}}{2\left(1+|z|^{2}\right) Q_{1}}\left(|0,0\rangle-e^{i 2 \phi}|1,1\rangle\right) .
$$

The success probability of this projection is

$$
Q_{1}^{2}=\frac{1+|z|^{4}+4|z|^{2} \cos ^{2} \varphi+\left(z^{2} e^{i 2 \varphi}+\text { c.c. }\right)}{2\left(1+|z|^{2}\right)^{2}} .
$$

Afterwards, a projection $P_{|0\rangle}$ onto the ground state of atom $B$ is implemented, leaving atom $A$ in the state

$$
-\frac{z e^{i \phi} \cos \varphi}{\left(1+|z|^{2}\right) Q_{1} Q_{2}}|1\rangle-\frac{e^{-i \varphi}+z^{2} e^{i \varphi}}{2\left(1+|z|^{2}\right) Q_{1} Q_{2}}|0\rangle .
$$

This event occurs with success probability $Q_{2}^{2}=1 / 2$. The overall success probability of the postselections is then given by

$$
P_{\mathrm{s}}=Q_{2}^{2} Q_{1}^{2}=Q_{1}^{2} / 2 \geqslant \frac{\cos ^{2} \varphi}{4}
$$

The last inequality follows from analyzing Eq. (1) and noting that $Q_{1}$ attains its minimum value when $|z|^{2}=1$ and $\operatorname{Re}\left[z^{2} e^{i 2 \varphi}\right]=-1$. Up to normalization the final state is given by

$$
|0\rangle+\frac{2 z \cos \varphi}{e^{-i \varphi}+z^{2} e^{i \varphi}} e^{i \phi}|1\rangle .
$$

By iterating this procedure we attain a scheme implementing the following quantum map for the $(n+1)$ th step:

$$
\frac{|0\rangle+f_{\varphi}^{n}(z) e^{i \phi}|1\rangle}{\sqrt{1+\left|f_{\varphi}^{n}(z)\right|^{2}}} \rightarrow \frac{|0\rangle+f_{\varphi}^{n+1}(z) e^{i \phi}|1\rangle}{\sqrt{1+\left|f_{\varphi}^{n+1}(z)\right|^{2}}},
$$

with the complex functions

$$
\begin{aligned}
f_{\varphi}(z) & =\frac{2 z \cos \varphi}{e^{-i \varphi}+z^{2} e^{i \varphi}}, \\
f_{\varphi}^{n+1}(z) & =f_{\varphi}\left(f_{\varphi}^{n}(z)\right), \quad f_{\varphi}^{0}(z)=z .
\end{aligned}
$$

The map is independent of the parameter $\phi$, as one can note that the phase factor $e^{i \phi}$ appears in the probability amplitude of state $|1\rangle$ in the same manner as in the initial state $\left|\psi_{0}\right\rangle$ of Eq. (23).

We note that the iteration of the map involves repeated action of the protocol on an ensemble of atoms. The protocol acts on a pair of identically prepared atoms from the ensemble and prepares one atom probabilistically. The other atom becomes useless from the point of view of the protocol as a result of the projective measurement on it. After acting on all the atoms of the ensemble, one arrives at a smaller ensemble less than one half the size. Rapid downscaling of the ensemble size is an unavoidable condition for any quantum dynamics truly sensitive to initial conditions [13]. In practice, realizing many steps of the protocol would require an exponentially large initial ensemble which would not be realistic. Another practical aspect is that employing more than one cavity would be challenging with today's experimental possibilities. On the other hand, as we will demonstrate in the next sections, already a few steps can be enough to make highly overlapping initial quantum states almost orthogonal. Furthermore, we will outline an experimental proposal in Sec. VI with currently available technology by applying an optical conveyor belt and a single cavity.

\section{BASIC PROPERTIES OF THE NONLINEAR MAP}

The dynamics within the approximations we have made is fully described by the iterative complex function in Eq. (31). This is a quadratic rational map [27], similar to the maps occurring in the measurement-induced nonlinear quantum dynamical schemes first described in $[5,11,13]$. In the following, we first carry out an analysis of the general properties of the iterated map $f_{\varphi}$ of Eq. (31) by using concepts from the theory of complex dynamical maps [28]. Then we compare its behavior to the numerical solution of the complete iterated dynamics based on the Hamiltonian of Eq. (1) and the subsequent selective measurements. 


\section{A. Stable cycles}

The periodic orbits or fixed cycles of the map $f_{\varphi}$ can be determined from the relation $f_{\varphi}^{n}(z)=z$. The one-cycles or fixed points as well as the two-cycles can be determined analytically. For $n=1$ we find

$$
z_{j}^{(1)}=j, \quad j \in\{-1,0,1\} .
$$

For $n=2$, in addition to the above one-cycles, one can find two more points which are transformed into each other by $f_{\varphi}$. These form the single nontrivial two-cycle

$$
z_{k}^{(2)}=(-1)^{k} i \sqrt{1+2 e^{-2 i \varphi}}, \quad k \in\{1,2\} .
$$

The stability of the fixed cycles can be checked by calculating the multiplier $\lambda=\left(f_{\varphi}^{n}\right)^{\prime}\left(z_{j}\right)=f_{\varphi}^{\prime}\left(z_{1}\right) f_{\varphi}^{\prime}\left(z_{2}\right) \cdots f_{\varphi}^{\prime}\left(z_{n}\right)$. A fixed cycle is repelling, neutral, attractive, or superattractive if $|\lambda|>1,|\lambda|=1,|\lambda|<1$, or $|\lambda|=0$, respectively. Such an analysis can be carried out analytically for the one- and two-cycles; however, for $n \geqslant 2$ it is a nontrivial task. The analysis of the multipliers shows that for each of the one-cycles there are certain parameter regions where they are attractive. On the other hand, the two-cycle given by Eq. (33) is repelling for any value of $\varphi$.

For the determination of the longer $(n \geqslant 3)$ attractive cycles we can use the method based on the iteration of the critical points of the map. The critical points of $f_{\varphi}$ are those which solve the equation $f_{\varphi}^{\prime}(z)=0$. In this case, there are two critical points:

$$
z_{c \pm}= \pm e^{-i \varphi}
$$

A general theorem on iterated rational polynomial maps states that a rational map of degree $d$ can have at most $2 d-2$ attractive cycles. Following the orbits of the critical points, one can find all stable cycles of the iterated map (in this case at most 2).

Figure 2 shows where, according to the analytical calculations, the one-cycles are superattractive (dots), attractive (lines), and neutral (circles) as a function of the parameter $\varphi$. The numerical iteration of the critical points in the regions between the neutral one-cycles shows that there are two different attractive four-cycles (orange lines) and a single six-cycle (green lines) close to the two ends of the regions. The

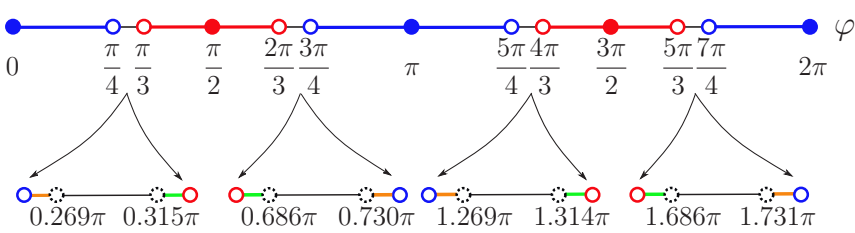

FIG. 2. Stability of the fixed cycles of $f_{\varphi}$ as a function of the parameter $\varphi$. Blue corresponds to the one-cycles $z^{(1)}= \pm 1$, red corresponds to $z^{(1)}=0$. Dots, lines, and circles represent superattractive, attractive, and neutral cycles, respectively. Numerical investigation of the enlarged regions between the neutral one-cycles shows two different attractive four-cycles (orange lines) and a single attractive six-cycle (green lines) close to the two ends of the region. The central part of the enlarged regions contains "islands" of attractive $n \geqslant 60$ cycles. The dotted circles indicate that is hard to identify the border of different regions. actual $z$ values belonging to the attractive four- and six-cycles depend on the parameter $\varphi$. In between these regions, it is numerically hard to rule out the existence of very long stable periodic orbits. The precision of our numerical simulation made it possible to identify a few "islands" of attractive fixed cycles of $n \geqslant 60$. The remaining part of this region may belong to maps without any stable periodic orbit, which means that all initial states belong to the Julia set. The dotted circles indicate that the border between different regions is hard to determine numerically, which is an indication of the fractal nature of the regions. Let us note that for $\varphi=\pi / 2$ and $3 \pi / 2$ the map is actually not a genuine complex map since $f_{\varphi} \equiv 0$ in these cases.

\section{B. Nature of the iterated map}

The fractal nature of the map is more apparent when one determines the Julia set of $f_{\varphi}$, i.e., the set of points which do not converge to an attractive cycle for a given $\varphi$. One way of numerically finding the points belonging to the Julia set is backwards iterating the map starting from a point which is an element of a repelling cycle of the map. We show in Fig. 3 the Julia set of $f_{\varphi}$ for $\varphi=1.666 \pi$. In this case, the Julia set is a totally disconnected set; all other initial points converge to the single attractive cycle $z=0$, or, physically speaking, to the state $|0\rangle$. The analysis of the orbits of the critical points reveals important properties of the Julia set. In this case both critical points converge to the same attractive fixed point; consequently, the Julia set is totally disconnected, similar to the well-known Cantor set [27]. Another important case is when the two critical points converge to two distinct fixed points; then the Julia set is connected. This case is illustrated by the map at parameter value $\varphi=0.95 \pi / 4$ shown in Fig. 4. For quadratic rational maps a general theorem ensures that the Julia set is either totally disconnected or connected [28].

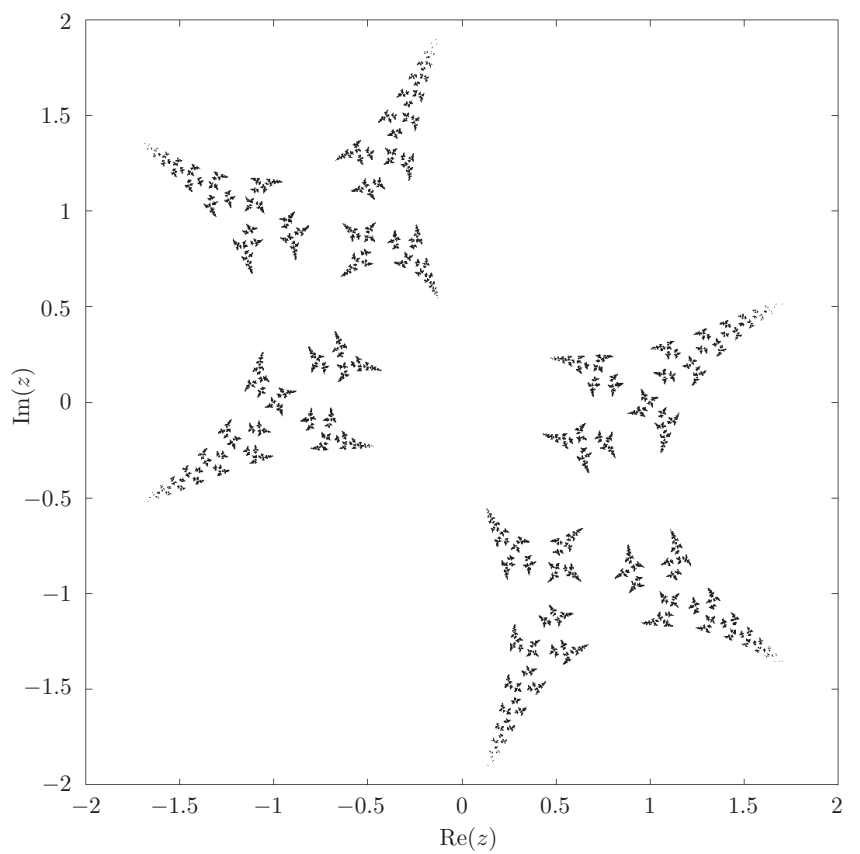

FIG. 3. The Julia set of the map $f_{\varphi}$ for $\varphi=1.666 \pi$. 

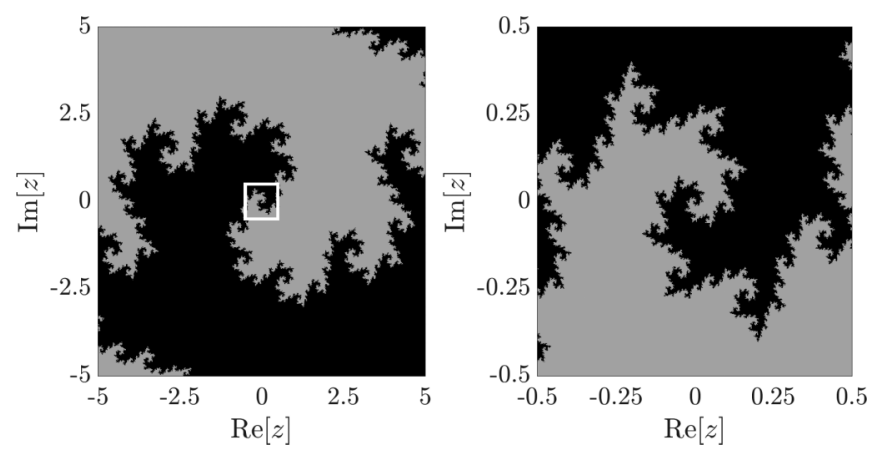

FIG. 4. Complex plane after 97 iterations of the map in Eq. (30) for $\varphi=0.95 \pi / 4$. Two amplification levels are shown, confirming the fractal structure of the Julia set separating the regions whose points converge to the attractive fixed points 1 (gray) and -1 (black). The region indicated by the square in the middle of the left plot is magnified in the right plot.

\section{Iteration of the complete dynamics}

In order to investigate the real performance of the two-atom Tavis-Cummings model without the approximations of Sec. II, we compute a numerically exact version of the operator $\hat{M}$ in Eq. (20). The matrix elements are evaluated as

$$
M_{j, k}=\left\langle\alpha\left|\left\langle e_{j}\left|e^{-i \hat{H} t / \hbar}\right| e_{k}\right\rangle\right| \alpha\right\rangle,
$$

where we considered the atomic basis $\left|e_{j}\right\rangle \in$ $\{|1,1\rangle,|1,0\rangle,|0,1\rangle,|0,0\rangle\}$. The interaction time $t$ and coupling strength $g$ satisfy the relation $g t=\pi \sqrt{\bar{n}} / 2$. Each iteration of the map is then evaluated by renormalizing the outcome $\left\langle\left. 0\right|_{B} \hat{M} \hat{U}_{B} \mid \Psi_{0}^{\text {at }}\right\rangle$ for qubit $A$. In Fig. 5 we plotted the real part of the 97th iteration for two different values of the mean photon number $\bar{n}$, namely, 100 and 10 . With precision of two (one) decimal places the two fixed points also converge to +1 and -1 in the case of $\bar{n}=100(\bar{n}=10)$. Both figures reveal a fractal structure which resembles more the ideal case for larger values of $\bar{n}$.

\section{APPLICATION OF THE PROTOCOL FOR STATE DISCRIMINATION}

The number of atoms needed by a protocol based on a nonlinear transformation grows exponentially with the number
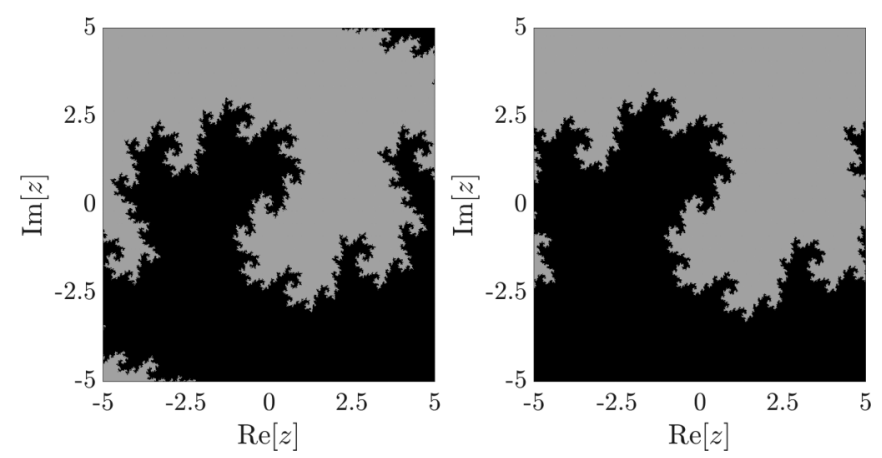

FIG. 5. The same as the left panel of Fig. 4 for the numerically exact quantum map and two values of the mean photon number $\bar{n}$ : 100 (left) and 10 (right). of iterations even in an ideal case, which follows from the quantum magnification bound [13]. In a realistic experiment, one can expect that only a few steps of the iteration can be carried out. On the other hand, a useful aspect of nonlinear quantum state transformations is that small initial differences between two similar quantum states can be amplified, enabling us to distinguish them, realizing a Schrödinger microscope [14]. Nonlinear quantum state transformations in an ideal case saturate the quantum magnification bound [13], thereby providing an optimal quantum state discrimination protocol, according to Helstrom [1]. Here we show that our protocol provides a practical state discrimination procedure, transforming initially very close states into almost perfectly orthogonal ones in as few as three steps.

In the simple case when $\varphi=0$, the nonlinear map reads $f_{\varphi=0}=2 z /\left(z^{2}+1\right)$, and the unitary of Eq. (24) is the wellknown $Z$ gate. In Fig. 6 we show the plane of initial states colored according to the number of iterations needed to reach either of the fixed points, 1 or -1 , with a precision of 0.1 . Complex numbers with a positive (negative) real part converge to the fixed point $1(-1)$. The two regions are separated by the Julia set of the map, which is indicated by the yellow region in the figure, coinciding with the imaginary axis. If we choose two initial quantum states close to each other in the form of Eq. (23) with $z_{1}=-0.2$ and $z_{2}=0.2$ (with an overlap close to unity $\left.\left|\left\langle\psi_{0}^{1} \mid \psi_{0}^{2}\right\rangle\right| \sim 0.92\right)$, then the two states will become almost orthogonal (with a scalar product of $\sim 0.08$ ) after three steps of the iteration.

The overlap of the above-mentioned two initial states converges fast to zero, as we show in Fig. 7. To account for possible imperfections in the preparation of the initial states we assumed a Gaussian uncertainty with a standard deviation of $\sigma=0.03$ in both the real and imaginary parts of the initial values $z_{1}=-0.2$ and $z_{2}=0.2$. We note that this value of $\sigma$ ensures that we sample from a distribution of quantum states which have either a positive or negative real part of the amplitude of state $|1\rangle$. Figure 7 (a) shows that due to the nonlinear transformation the resulting uncertainty (represented by the error bars) in the initial value of the scalar product grows in the first and second steps but then decreases and eventually becomes much smaller than its initial value

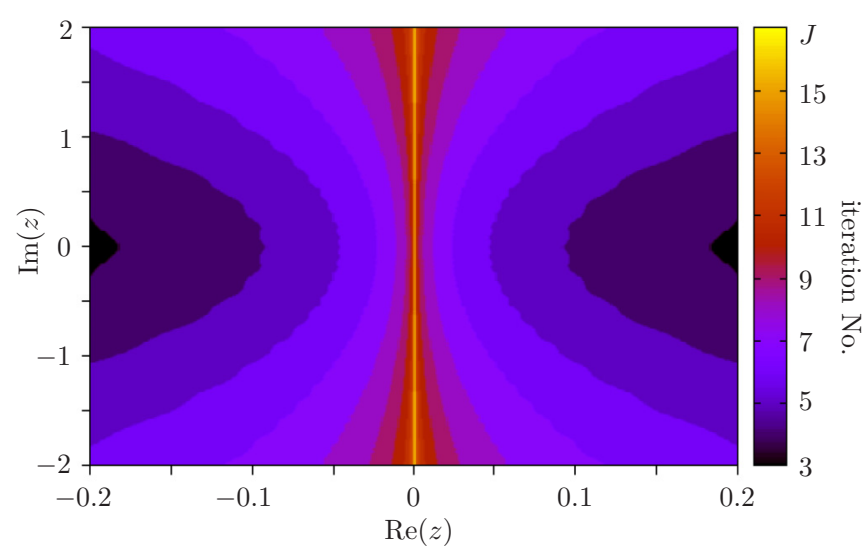

FIG. 6. The plane of initial states colored according to the number of iterations needed for a complex number $z$ to reach either of the fixed points, 1 or -1 , with a precision of 0.1 . 

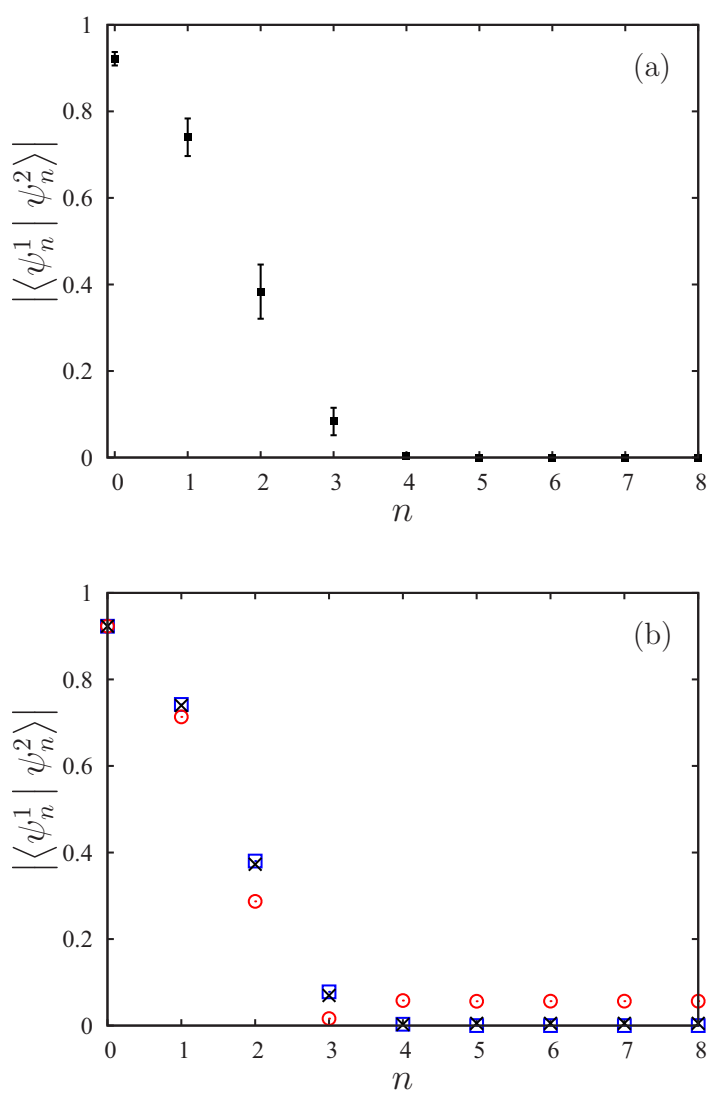

FIG. 7. (a) The overlap of the states $\left|\psi_{0}^{1}\right\rangle=0.98(|0\rangle-0.2|1\rangle)$ and $\left|\psi_{0}^{2}\right\rangle=0.98(|0\rangle+0.2|1\rangle)$ after $n$ iterations of the ideal map, when there is an uncertainty described by a Gaussian distribution of standard deviation $\sigma=0.03$ around both the real and imaginary parts of the initial values $z_{1}=-0.2$ and $z_{2}=0.2$. The error bars represent the rms deviation from the mean (black squares) of the possible values of the scalar product. (b) The overlap of the states after $n$ iterations of the ideal map (blue squares) and the complete map with a mean photon number of 10 (red circles) and 100 (black crosses).

(the error bars cannot be seen at the resolution of the figure for $n \geqslant 4)$. Thus our procedure effectively discriminates between two different phases of small excitation amplitudes of the atoms. The evolution of the overlap of the above two initial states is not modified significantly when using the complete solution for the map, as can be seen in Fig. 7(b). Mean photon numbers of $\bar{n}=10$ and $\bar{n}=100$ lead to behavior essentially similar to that of the idealized map (31). Interestingly, the low-photon-number case leads to a faster decrease in the overlap during the first few steps of the iteration but then converges to a larger value compared to the ideal map.

\section{EXPERIMENTAL CONSIDERATIONS}

Our basic protocol involves atomic and photonic postselection, and therefore there is always a finite probability of failure. This means that in order to implement several iterations of the map, one requires several copies of the initial qubit pair. The procedure explained in Sec. III has to be applied to every single copy of the ensemble. The number $N$ of qubit pairs required to achieve $n$ iterations can be bounded from below by taking

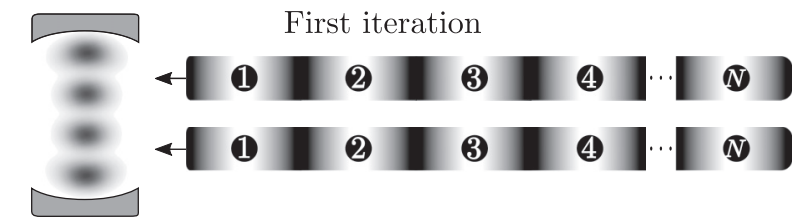

Second iteration

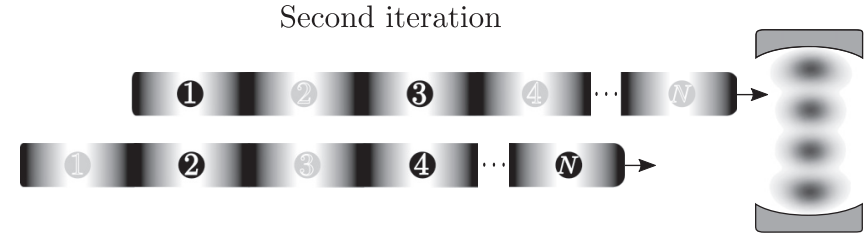

FIG. 8. Possible implementation of the protocol using neutral atoms coherently transported using optical conveyor belts.

into account the success probability $P_{\mathrm{s}}$ in (28). In addition, one has to take into account that half of the atoms in the ensemble are lost after being measured. Therefore the number of pairs scales exponentially as $N=\left(2 / P_{\mathrm{S}}\right)^{n}=\left(8 / \cos ^{2} \varphi\right)^{n}$.

At first glance one would naively consider the use of $N$ optical cavities for $N$ atomic pairs. However, there is another simpler solution motivated by current experimental implementations $[29,30]$, where a standing-wave dipole trap, or "optical conveyor belt", is used to coherently transport neutral atoms into an optical resonator.

Using this setting, two conveyor belts are required to transport atoms into the cavity. In the initial stage, $N$ atoms are prepared in the minima of the two optical traps and are aligned as depicted in Fig. 8. For convenience, we number the atoms from left to right. The unitary gate $\hat{U}_{\varphi}$ is applied at this preparatory stage to atoms labeled with an even (odd) number in the upper (lower) conveyor belt; we call them marked atoms. The two conveyor belts are moved forwards into the direction of the cavity until the first pair reaches the other side of the cavity. Then, the conveyor belts stop in order to allow the measurement of the first marked atom and the field inside the cavity. Afterwards, the cavity is reset to the state $|\alpha\rangle$, and the conveyor belts move again, repeating the process. After all atoms have interacted with the cavity, the marked atoms are blacklisted as they are no longer useful. They are depicted in gray in Fig. 8. In order to pair only the useful atoms, the lower conveyor belt is shifted one period to the left, leaving the first marked atom without a partner. In this way, the potentially successfully prepared atoms are aligned. The process is repeated with both conveyor belts moving to the opposite side to start the second iteration. In the aforementioned implementation of the second iteration we have ignored the possibility of failure in the postselection. In order to overcome this problem, one has to keep track of successfully prepared atoms and then shift the conveyor belts in order to align useful pairs before transporting them into the cavity.

Finally, it is worth noting that for the sake of simplicity we have considered only the dynamics generated by the Hamiltonian $\hat{H}$ [see Eq. (1)] in the interaction picture with respect to the reference Hamiltonian $\hat{H}_{0}$ [see Eq. (2)]. In the Schrödinger picture, or laboratory reference frame, the only differences are due to the free time evolution resulting from the Hamiltonian of Eq. (2). They lead to a relative phase 
in the atomic state, and they have to be taken into account in the field measurements [compare with Eq. (18), for example]. The one-atom state after a step of the protocol and up to normalization can be written as $|0\rangle+f_{\varphi}(z) e^{i \phi-i \omega\left(t+t_{1}+t_{2}\right)}|1\rangle$. Here we have considered a free evolution with time $t_{1}\left(t_{2}\right)$ before (after) the interaction which takes place for a time $t$. To prove this it suffices to note that $\hat{H}$ commutes with $\hat{H}_{0}$, and therefore one can split the evolution operator in the Schrödinger picture as

$$
\begin{aligned}
\hat{\mathcal{U}} & =e^{-i \hat{H}_{0} t_{2} / \hbar} e^{-i\left(\hat{H}+\hat{H}_{0}\right) t / \hbar} e^{-i \hat{H}_{0} t_{1} / \hbar} \\
& =e^{-i \hat{H}_{0}\left(t+t_{1}+t_{2}\right) / \hbar} e^{-i \hat{H} t / \hbar} .
\end{aligned}
$$

After the evolution, the field and atom $B$ are projected into pure states, yielding for atom $A$ an evolution operator $\exp \left[-i \omega|1\rangle\left\langle\left. 1\right|_{A}\left(t+t_{1}+t_{2}\right)\right]\right.$ which generates the mentioned phase. In order to keep the same form of the map in Eq. (31), one could adjust the times in such a way that $t+t_{1}+t_{2}=$ $2 \pi / \omega$. Alternatively, one could eliminate this phase by driving the atoms with a classical electromagnetic field in a way similar to what we proposed to implement the gate $\hat{U}_{\varphi}$ in Eq. (24).

\section{CONCLUSION}

We have proposed a nonlinear map of qubit states in a cavity quantum electrodynamical scenario where the qubits are encoded in two-level atoms. The core step requires the interaction of two equally prepared atoms with the field inside an optical resonator according to the Tavis-Cummings model. By subsequent field detection and selective measurement of one of the atoms, the unmeasured atom is postselected into a state nonlinearly depending on its initial state.

From a mathematical point of view, we have studied the complex function describing this mapping of pure qubit states, where we have exploited the fact that any pure state of a qubit can be described by a complex parameter. We have performed an analysis of stable cycles under the iteration of the function and studied the behavior in the complex plane. In particular, we have numerically investigated the Julia set, which changes from connected to disconnected for different parameters of the system. Thus, our study offers a demonstration of chaotic behavior in a quantum-mechanical setting involving sequences of unitary transformations and postselective measurements. From a physical perspective, we have proposed the realization of this scheme using an ensemble of equally prepared atoms in two optical conveyor belts that are coherently transported and interact in pairs with a single optical resonator. We have estimated the number of atoms required for each iteration of the protocol, taking into account the success probability of the measurements involved. Although possible realizations of this nonlinear qubit map require cutting-edge quantum technological developments, such as optical conveyor belts and controlled two-qubit interactions with a single-mode radiation field, in view of the rapid experimental advances in cavity quantum electrodynamics its realization is within reach of current technology.

The presented scheme provides an alternative approach to already established quantum state discrimination protocols [31]. We suggested an effective implementation of the Schrödinger microscope in which two initially close pure quantum states can be discriminated by amplifying the distance between them and thus effectively orthogonalizing them. We have shown that initial states of the two-level atoms with high overlap will become almost perfectly orthogonal by a few iterations of the scheme. Let us note that the orthogonalization procedure has a slightly different flavor than previous quantum state discrimination procedures. First, it is deterministic in the sense that there is a probability of success for the whole process, but then the resulting quantum state is fully determined by the initial state. Second, it does not directly measure the orthogonalized systems, but rather prepares them in a nondemolition sense, and therefore these systems can be used for further processing. Third, it is a purification process as well, which naturally accounts for initial noise, and effectively discriminates mixed nonorthogonal quantum states. Measurement-induced nonlinear evolution in quantum mechanics is a concept which could be used by other physical realizations of qubits to implement a Schrödinger microscope.

\section{ACKNOWLEDGMENTS}

This work was supported by the Hungarian Academy of Sciences (Lendület Program, LP2011-016) and the National Research, Development and Innovation Office (K115624, NN109651, PD120975), and by the Deutscher Akademischer Austauschdienst (MÖB-DAAD Project No. 65049). O.K. acknowledges support from the János Bolyai Research Scholarship of the Hungarian Academy of Sciences.
[1] C. W. Helstrom, Quantum Detection and Estimation Theory (Academic Press, New York, 1976).

[2] I. D. Ivanovic, Phys. Lett. A 123, 257 (1987).

[3] D. Dieks, Phys. Lett. A 126, 303 (1988).

[4] A. Peres, Phys. Lett. A 128, 19 (1988).

[5] H. Bechmann-Pasquinucci, B. Huttner, and N. Gisin, Phys. Lett. A 242, 198 (1998).

[6] C. H. Bennett, G. Brassard, S. Popescu, B. Schumacher, J. A. Smolin, and W. K. Wootters, Phys. Rev. Lett. 76, 722 (1996); 78, 2031 (1997).

[7] D. Deutsch, A. Ekert, R. Jozsa, C. Macchiavello, S. Popescu, and A. Sanpera, Phys. Rev. Lett. 77, 2818 (1996); 80, 2022 (1998).
[8] C. Macchiavello, Phys. Lett. A 246, 385 (1998).

[9] G. Alber, A. Delgado, N. Gisin, and I. Jex, J. Phys. A 34, 8821 (2001).

[10] J. M. Torres and J. Z. Bernád, Phys. Rev. A 94, 052329 (2016).

[11] T. Kiss, I. Jex, G. Alber, and S. Vymětal, Phys. Rev. A 74, 040301(R) (2006).

[12] T. Kiss, S. Vymetal, L. D. Toth, A. Gabris, I. Jex, and G. Alber, Phys. Rev. Lett. 107, 100501 (2011).

[13] A. Gilyén, T. Kiss, and I. Jex, Sci. Rep. 6, 20076 (2016).

[14] S. Lloyd and J.-J. E. Slotine, Phys. Rev. A 62, 012307 (2000).

[15] R. B. M. Clarke, A. Chefles, S. M. Barnett, and E. Riis, Phys. Rev. A 63, 040305 (2001). 
[16] A. C. Dada, E. Andersson, M. L. Jones, V. M. Kendon, and M. S. Everitt, Phys. Rev. A 83, 042339 (2011).

[17] G. Waldherr, A. C. Dada, Ph. Neumann, F. Jelezko, E. Andersson, and J. Wrachtrup, Phys. Rev. Lett. 109, 180501 (2012).

[18] R. Reimann, W. Alt, T. Kampschulte, T. Macha, L. Ratschbacher, N. Thau, S. Yoon, and D. Meschede, Phys. Rev. Lett. 114, 023601 (2015).

[19] A. Neuzner, M. Körber, O. Morin, S. Ritter, and G. Rempe, Nat. Photonics 10, 303 (2016).

[20] M. Tavis and F. W. Cummings, Phys. Rev. 170, 379 (1968).

[21] J. M. Torres, J. Z. Bernád, and G. Alber, Phys. Rev. A 90, 012304 (2014).

[22] J. M. Torres, J. Z. Bernád, and G. Alber, Appl. Phys. B 122, 117 (2016).

[23] A. I. Lvovsky and M. G. Raymer, Rev. Mod. Phys. 81, 299 (2009).

[24] M. A. Nielsen and I. L. Chuang, Quantum Computation and Quantum Information (Cambridge University Press, Cambridge, 2000).
[25] D. Meschede and A. Rauschenbeutel, Adv. At. Mol. Opt. Phys. 53, 75 (2006).

[26] J. M. Raimond, M. Brune, and S. Haroche, Rev. Mod. Phys. 73, 565 (2001).

[27] J. Milnor and T. Lei, Exp. Math. 2, 37 (1993).

[28] J. Milnor, Dynamics in One Complex Variable, Annals of Mathematical Studies Vol. 1160 (Princeton University Press, Princeton, NJ, 2006).

[29] M. Khudaverdyan, W. Alt, I. Dotsenko, T. Kampschulte, K. Lenhard, A. Rauschenbeutel, S. Reick, K. Schrner, A. Widera, and D. Meschede, New J. Phys. 10, 073023 (2008).

[30] S. Brakhane, W. Alt, T. Kampschulte, M. Martinez-Dorantes, R. Reimann, S. Yoon, A. Widera, and D. Meschede, Phys. Rev. Lett. 109, 173601 (2012).

[31] J. A. Bergou, U. Herzog, and M. Hillery, in Quantum State Estimation, edited by M. G. A. Paris and J. Řeháček, Lecture Notes in Physics Vol. 649 (Springer, Berlin, 2004), pp. 417466. 